\title{
A low cost rapid urease test to detect Helicobacter pylori infection in resource limited settings
}

\author{
S M Buharideen ${ }^{1}$, S Wijetunge ${ }^{1}$, R Kotakadeniya ${ }^{2}$, K B Galketiya ${ }^{2}$, B Samarasinghe ${ }^{2}$, A Dharmapala ${ }^{2}$, \\ F Noordeen ${ }^{3}$
}

(Index words: Helicobacter pylori, one minute rapid urease test, one day rapid urease test, diagnosis)

\begin{abstract}
The aim of the study was to evaluate the suitability of a modified one minute rapid urease test (one day rapid urease test) as a low cost $H$. pylori detection method. A sample of 205 patients clinically suspected of having $H$. pylori infection was tested. One day rapid urease test and histology based $H$. pylori tests (the gold standard) were performed on endoscopic antral biopsies. There were 6 true positives, 191 true negatives, 8 false positives and zero false negatives. The sensitivity, specificity and positive (PPV) and negative predictive values (NPV) of the test were $100 \%, 96 \%, 42.9 \%$, and $100 \%$ respectively. The cost per patient was 0.3 US\$. High sensitivity, specificity and NPV, low cost and simplicity of method were the advantages of the test and the main limitation was low PPV. Hence, one day rapid urease test can be considered as a suitable low cost method to detect $H$. pylori infection in resource limited settings.
\end{abstract}

Ceylon Medical Journal 2015; 60: 21-23

\section{Introduction}

Empirical treatment of patients with suspected Helicobacter pylori gastritis with triple therapy is discouraged because of emergence of antibiotic resistance and test and treat strategy has been advocated as a proven management strategy $[1,2]$. However, in developing countries, lack of an affordable $H$. pylori diagnostic test is a major limitation in implementing this strategy. H. pylori detection methods such as histology, culture and urease breath test require expensive infrastructure and trained personnel. Although commercially available methods such as CLO test, stool antigen test and serology are easy to carry out, they are too expensive for routine use in developing countries. Therefore, an inexpensive H. pylori detection method with reasonable validity is required for use in these countries.

One minute rapid urease test described by Arvind et $a l$ is an inexpensive test carried out using locally prepared solutions and has a sensitivity and specificity of $91 \%$ and $100 \%$ respectively [3]. We conducted the following study to evaluate the suitability of a modified version of one minute rapid urease test (one day rapid urease test) as a $H$. pylori detection method in patients undergoing upper gastrointestinal endoscopy, in resource limited settings.

\section{Methods}

This cross sectional study was conducted on 205 patients who underwent upper gastrointestinal endoscopy and were clinically and endoscopically suspected of having $H$. pylori gastritis. Study was conducted at the Teaching Hospital, Peradeniya, Sri Lanka from 2012 to 2013. These patients had dyspeptic symptoms and endoscopic evidence of antral mucosal inflammation such as erythema, erosions or peptic ulcers. None of them had been treated with triple therapy during the past one year.

All patients underwent a standard biopsy protocol which included three biopsies from the antral mucosal lesion (erythema, erosions or ulcer). One biopsy was immediately utilised to perform one day rapid urease test in the endoscopy room and the rest were collected in $10 \%$ formalin solution for histology and immunohistochemistry. Formalin fixed and paraffin embedded tissue sections were stained with haematoxylin and eosin and toluidine blue. H. pylori specific immunohistochemistry (DAKO B0471) was performed using peroxidase method. Diagnostic efficacy of one day rapid urease test was evaluated by calculating sensitivity, specificity negative predictive value (NPV) and positive predictive value (PPV) using histology based methods as the gold standard. Positive results with one or more of the three histology based tests were considered as evidence for presence of $H$. pylori infection.

The procedure for the original one minute rapid urease test described by Arvind et al is as follows, "an antral mucosal biopsy is placed immediately into a capped Eppendorf tube containing $05 \mathrm{ml}$ freshly prepared $10 \%$ urea $(\mathrm{w} / \mathrm{v})$ in deionised water at a $\mathrm{pH}$ of around 6.8 , to

Departments of ${ }^{1}$ Pathology, ${ }^{2}$ Surgery and ${ }^{3}$ Microbiology, Faculty of Medicine, University of Peradeniya, Sri Lanka.

Correspondence: SW, e-mail: <suwijetunge@gmail.com>. Received 17 April and revised version accepted 7 November 2014. Competing interests: none declared. 
which two drops of $1 \%$ phenol red (free acid) is added as a $\mathrm{pH}$ indicator [3]. A positive result is recorded if there is a colour change from yellow to pink within the first minute". We followed the same procedure with the following modifications. 1. Instead of freshly prepared $10 \%$ urea solution, we prepared a stock of urea solution once a week and stored in a domestic type refrigerator and aliquots of solution were brought to the room temperature before use; 2 . Distilled water was used instead of deionised water; 3. Solution was observed for a colour change up to 24 hours and interpreted as follows: an obvious colour change from yellow to pink involving the entire test solution within 24 hours was regarded as a positive reaction. Weak colour changes such as, a subtle colour change giving rise to a cloudy appearance or presence of a pinkish smoke like line emanating from the biopsy and no colour change were regarded as negative reactions.

Venous blood was drawn from each patient to screen for presence of anti $H$. pylori antibodies using a qualitative immunochromatographic method (SD bioline H. pylori).

\section{Results}

The mean age of the sample was $56.9 \pm 14$ years and $56.6 \%$ were males. One day rapid urease test was positive in 14 cases and in 6 at least one histology based method demonstrated presence of $H$. pylori organisms (Table 1). Accordingly, one day rapid urease test had $100 \%$ sensitivity (95\% CI $0.54-1.0)$; $96 \%$ specificity $(95 \%$ CI $0.92-0.98)$; $42.9 \%$ PPV (95\% CI $0.18-0.71$ ), and 100\% NPV (95\% CI 0.98 - 1). Accuracy of the test was $96.1 \%$ and $2.9 \%$ of the sample were $H$. pylori positive.

Of the 14 one day rapid urease test positive cases, 12 became positive within one minute and the two showed positivity later, one within one hour and the other within

\section{Table 1. Comparison of one day rapid urease test with the gold standard method}

\begin{tabular}{lccc}
\hline & $\begin{array}{c}\text { H. pylori } \\
\text { positive }\end{array}$ & $\begin{array}{c}\text { H. pylori } \\
\text { negative }\end{array}$ & Total \\
\hline $\begin{array}{l}\text { One day rapid } \\
\text { urease test positive }\end{array}$ & 6 & 8 & 14 \\
$\begin{array}{l}\text { One day rapid } \\
\text { urease test negative }\end{array}$ & 0 & 191 & 191 \\
Total & 6 & 199 & 205 \\
\hline
\end{tabular}

24 hours. All these were true positives. Twenty one cases showed a weak colour change and all of them were true negatives. In 10/205 (4.9\%) anti $H$. pylori antibodies were present. Cost of the test per patient was about 30 Sri Lankan Rupees (0.3 US dollars).

\section{Discussion}

One day rapid urease test has a high sensitivity
$(100 \%)$ and a reasonable specificity (96\%) in detecting H. pylori infection. The original version had a sensitivity of $91 \%$ and a specificity of $100 \%$ [3].

Superior sensitivity and the NPV of one day rapid urease test are due to observation of the test up to 24 hours; the two late positives were true positives. Therefore, we recommend observing the solution up to 24 hours as in CLO test. Furthermore, absence of true positives in the 21 cases with weak colour changes justify treating weak reactions as negative. Interpretation of weak colour changes as positive would have reduced the specificity of the test to $85.4 \%$.

The main limitation of the study was the low prevalence of $H$. pylori infection in the sample tested $(2.9 \%)$ which can influence the predictive values of the detection methods. This could explain the low PPV $(42.9 \%)$ of the present test. The low seroprevalence of anti H. pylori antibodies $(4.9 \%)$ confirms that the $H$. pylori prevalence in the sample is truly low and the study sample had low exposure to the organism. In contrast, with the one minute rapid urease test. PPV was $100 \%$ and the disease prevalence in the sample tested was $52.5 \%$.

Although the urea solution should ideally be prepared fresh, the authors of the original study had observed that pre-prepared frozen solutions could also be used by thawing just before use [4]. In our experience, storage of pre-prepared urea solution in the door compartment of a domestic type refrigerator up to 7 days did not significantly change the test efficacy. The optimal action of urease enzyme is at $45^{\circ} \mathrm{C}$ and therefore, it is important to bring the solution to room temperature before testing to prevent false negatives.

In conclusion, high sensitivity, specificity and NPV, low cost and simple methodology were the advantages of one day rapid urease test and the main limitation was low PPV. Hence, one day rapid urease test can be considered as a suitable low cost method to detect $H$. pylori infection in gastric biopsies in resource limited settings.

\section{Acknowledgements}

Authors acknowledge the financial assistance by the National Science Foundation of Sri Lanka (Grant No RG/2011/HS/11), technical assistance by Mrs. T. R. D. S. K. Tennakoon, Mrs. S. M. Wickramasingha and Mrs. N. Herath and the cooperation of the nursing staff of the Endoscopy Unit, Teaching Hospital, Peradeniya.

\section{Declaration of Interest}

There are no conflicts of interest. 


\section{References}

1. Chey WD, Wong BCY and the Practice Parameters Committee of the American College of Gastroenterology. American College of Gastroenterology Guideline on the Management of Helicobacter pylori Infection. Am J Gastroenterol 2007; 102: 1808-25.

2. Malfertheiner P, Megraud F, O'Morain CA, Atherton J, Axon AT, Bazzoli F, Gensini GF, Gisbert JP, Graham DY, Rokkas T, Omar EME, Kuipers EJ, The European Helicobacter study group. Management of Helicobacter pylori infection - the Maastricht IV/Florence Consensus Report. Gut 2012; 61: 646-64.
3. Arvind AS, Cook RS, Tabaqchali S, Farthing MJG. One minute endoscopy room test for Campylobacter pylori. Lancet 1988; 331: 704.

4. Thillainayagam AV, Arvind AS, Cook RS, Harrison IG, Tabaqchali S, Farthing MJG. Diagnostic efficiency of an ultra rapid endoscopy room test for Helicobacter pylori. Gut 1991; 32: 467-9. 